\title{
Le système SPOT et la qualité de ses images
}

\author{
M. Leroy
}

Centre National d'Etudes Spatiales, 18 av. Edouard Belin, F-31055 Toulouse cedex, France

\section{INTRODUCTION}

La télédétection des paysages terrestres à l'aide de capteurs spatiaux est un outil d'analyse performant des surfaces terrestres pour un ensemble varié d'applications : cartographie, inventaires urbains ou ruraux, classification et suivi de ressources renouvelables agricoles ou forestières, aménagement du territoire, examen de l'hydrographie des sols, bathymétrie des zones côtières, détection de structures géologiques, mesures du relief.

La filière de satellites SPOT d'observation de la Terre a été conçue pour répondre à ces multiples besoins. Le premier de ces satellites, SPOT 1, a été lancé le 22 février 1986 et a maintenant terminé sa phase d'exploitation après plus de quatre années de bons et loyaux services. Le satellite SPOT 2, pratiquement identique à SPOT 1, a été lancé le 22 janvier 1990, et est actuellement opérationnel. D'autres satellites de la filière SPOT sont en cours de préparation.

L'originalité du concept optique des instruments de SPOT tient à deux nouveautés importantes par rapport aux premiers satellites d'observation. D'une part, le balayage de l'image est obtenu en imageant une ligne de paysage sur une ligne de détecteurs photosensibles CCD, l'exploration de l'autre dimension du paysage étant assurée par le défilement du satellite. D'autre part, il existe une possibilité de dépointage latéral, perpendiculairement à la trace au sol du satellite, de l'axe optique de visée des instruments.

Les conséquences de ces caractéristiques de conception sur la qualité des images sont évidemment tout-à-fait significatives.

L'objet du présent article est de présenter dans ses grandes lignes le système SPOT, en mettant plus particulièrement l'accent sur les caractéristiques optiques de ses instruments, puis de décrire la qualité de ses images, ainsi que les principes d'étalonnage qui permettent de maintenir à un bon niveau la qualité des images durant la vie orbitale.

\section{LE SYSTEME SPOT}

\subsection{Fonctionnement général}

Le satellite SPOT contient deux instruments optiques appelés HRV (Haute Résolution dans le Visible) capables d'observer la Terre à 10 mètres de résolution dans le mode panchromatique ( $0.51-0.73$ microns), et à 20 mètres de résolution dans le mode multibandes [Réfs. 1,2$]$. Ce dernier mode contient 3 bandes spectrales, verte $(0.50-0.59$ microns), rouge $(0.61$ 0.68 microns) et proche infrarouge ( $0.79-0.89$ microns), dont le choix a été optimisé pour obtenir une discrimination maximale des différents types de cibles terrestres observables [Réf. 3], comme l'illustre la Figure 1.

La taille au sol des images est de $60 \mathrm{~km}$. Lorsque les deux instruments HRV1 et HRV2 travaillent en mode jumelé (Figure 2a) ils couvrent une bande de terrain dont la largeur avoisine $120 \mathrm{~km}$. L'axe optique de visée de chacun des instruments peut être déplacé perpendiculairement à la trace d'une valeur maximale de $\pm 27^{\circ}$, au moyen d'un miroir à 


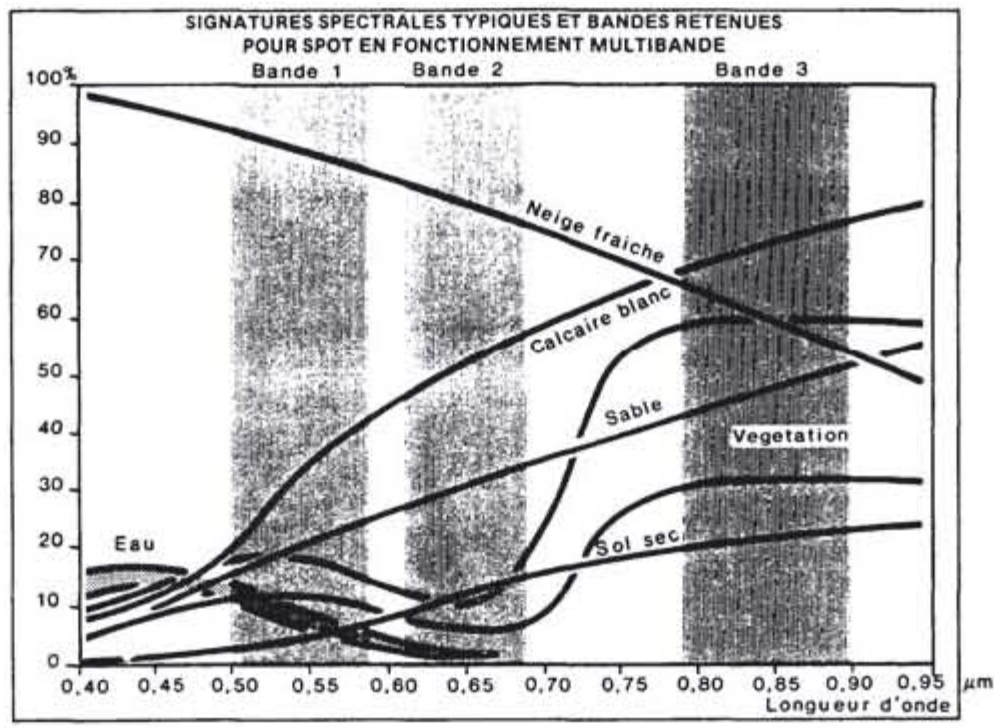

Figure 1 : Les trois bandes de longueur d'onde retenues pour SPOT (vert, rouge, proche infrarouge) avec une résolution au sol de $20 \mathrm{~m}$ ont été choisies en fonction des différentes utilisations de SPOT. Le schéma permet de situer la réflectance (\%) de quelques éléments végétaux ou minéraux typiques suivant la longueur d'onde du rayonnement incident.

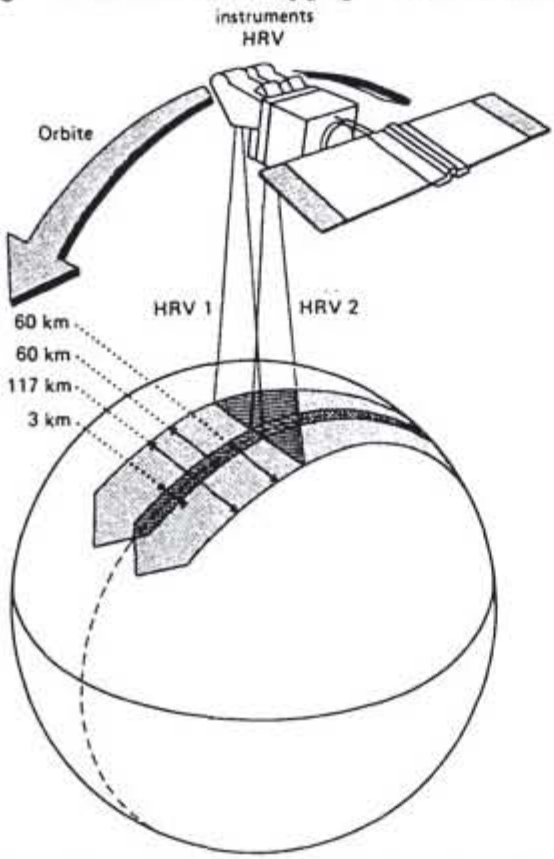

Figure 2a : Observation de bandes de terrain en visée verticale.

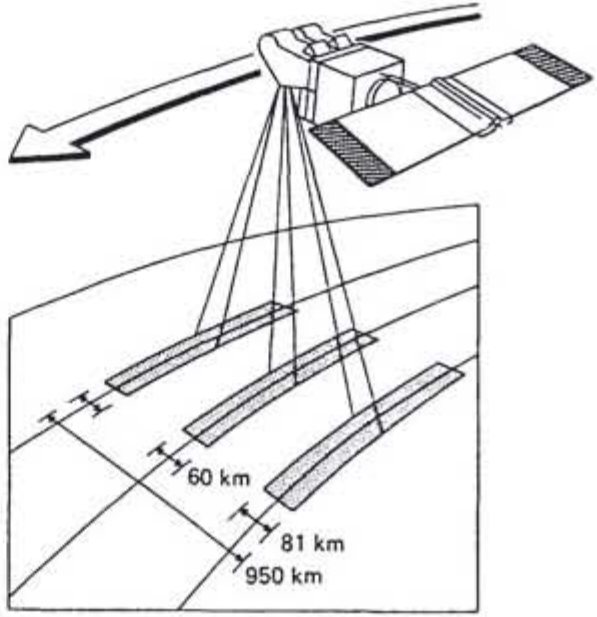

Figure 2b : Observation de bandes de terrain en visée verticale et en visée oblique. 
changement de visée (MCV). La largeur de terrain accessible par un instrument est alors de $950 \mathrm{~km}$ (Figure $2 \mathrm{~b}$ ).

Le cycle orbital du satellite, période de temps qui sépare deux passages au-dessus d'une même région, est de 26 jours.

Ceci signifie que si le satellite ne pouvait effectuer que des visées verticales, il faudrait attendre 26 jours pour réaliser deux observations d'une même région. La visée oblique permet d'augmenter sensiblement la fréquence des observations; par exemple, à la latitude de $45^{\circ}$, une même région peut être observée 12 fois pendant le cycle orbital.

Les visées obliques permettent en outre d'obtenir des couples d'images stéréoscopiques d'une même scène prises sous des angles différents lors des révolutions orbitales successives du

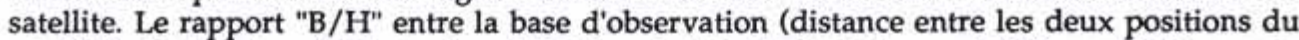
satellite) et la hauteur (altitude du satellite) peut atteindre 1. Cette possibilité, combinée avec la haute résolution des images panchromatiques, est particulièrement intéressante pour les applications cartographiques (perception du relief, géomorphologie, études hydrographiques).

\section{LE SATELLITE}

- Masse totale (début de vie)

$1830 \mathrm{~kg}$

- Générateur solaire (puissance disponible sur la partie éclairée de l'orbite) $1 \mathrm{~kW}$

- Envergure panneaux solaires $\quad 15,60 \mathrm{~m}$

- Altitude (à $45^{\circ}$ de latitude nord) $832 \mathrm{~km}$

- Période de révolution (valeur nominale) 101,4 min

- Dimension du corps principal $2 \times 2 \times 3,5 \mathrm{~m}$

- Capacité d'enregistrement $2 \times 22 \mathrm{mn}$

\section{L'INSTRUMENT HRV}

- Bande spectrale

mode multibande

XS1 : 0,50-0,59 microns

$\mathrm{XS} 2: 0,61-0,68$ microns

XS3 : $0,79-0,89$ microns

- Champ

- Dimension du pixel

4,13 degrés

$20 \times 20 \mathrm{~m}$

3000

$60 \mathrm{~km}$

- Largeur de bande observée mode panchromatique

$\mathrm{Pa}: 0,51-0,73$ microns

\section{4,13 degrés}

$10 \mathrm{~m} \times 10 \mathrm{~m}$

6000

$60 \mathrm{~km}$

\section{LIAISON IMAGE}

- Fréquence

- Rythme de transmission

- Fréquence de la balise (aide à la poursuite)

$8307 \mathrm{MHz}$

Tableau 1 - Quelques caractéristiques de SPOT 


\subsection{L'orbite}

L'orbite est choisie circulaire, à l'altitude quasi-constante de $830 \mathrm{~km}$, de façon à ce que les images aient les mêmes caractéristiques quel que soit le point observé, et quasi-polaire, de telle sorte que l'on obtienne des images de toutes les régions terrestres.

Pour pouvoir comparer des observations d'un point donné prises à des dates différentes, il est nécessaire que les conditions d'éclairement solaire soient proches d'une observation à l'autre. On ne peut rien contre les variations saisonnières de hauteur zénithale solaire. On peut par contre chercher à survoler un point donné toujours à la même heure solaire locale.

Cela est obtenu en choisissant une orbite héliosynchrone, c'est-à-dire une orbite dont la ligne des noeuds (intersection du plan d'orbite avec l'Equateur terrestre) fait exactement un tour en un an, comme la terre dans son mouvement autour du soleil. De la sorte, l'angle entre la direction du soleil et le plan d'orbite reste constant tout au long de l'année (Figure 3). La rotation de la ligne des noeuds, dont la vitesse est fonction de l'inclinaison de l'orbite et de son altitude résulte en fait d'une perturbation (par rapport aux lois de Kepler) due principalement à l'aplatissement terrestre. Pour une orbite circulaire à $830 \mathrm{~km}$, l'héliosynchronisme est obtenu pour une inclinaison de $98,7^{\circ}$.

De façon nominale, le satellite passe au noeud descendant (intersection de sa trace descendante avec l'Equateur) à $10 \mathrm{~h} 30$ du matin. Pour une latitude inférieure à $60^{\circ}$, l'heure locale varie faiblement avec la latitude et l'angle de dépointage du MCV.
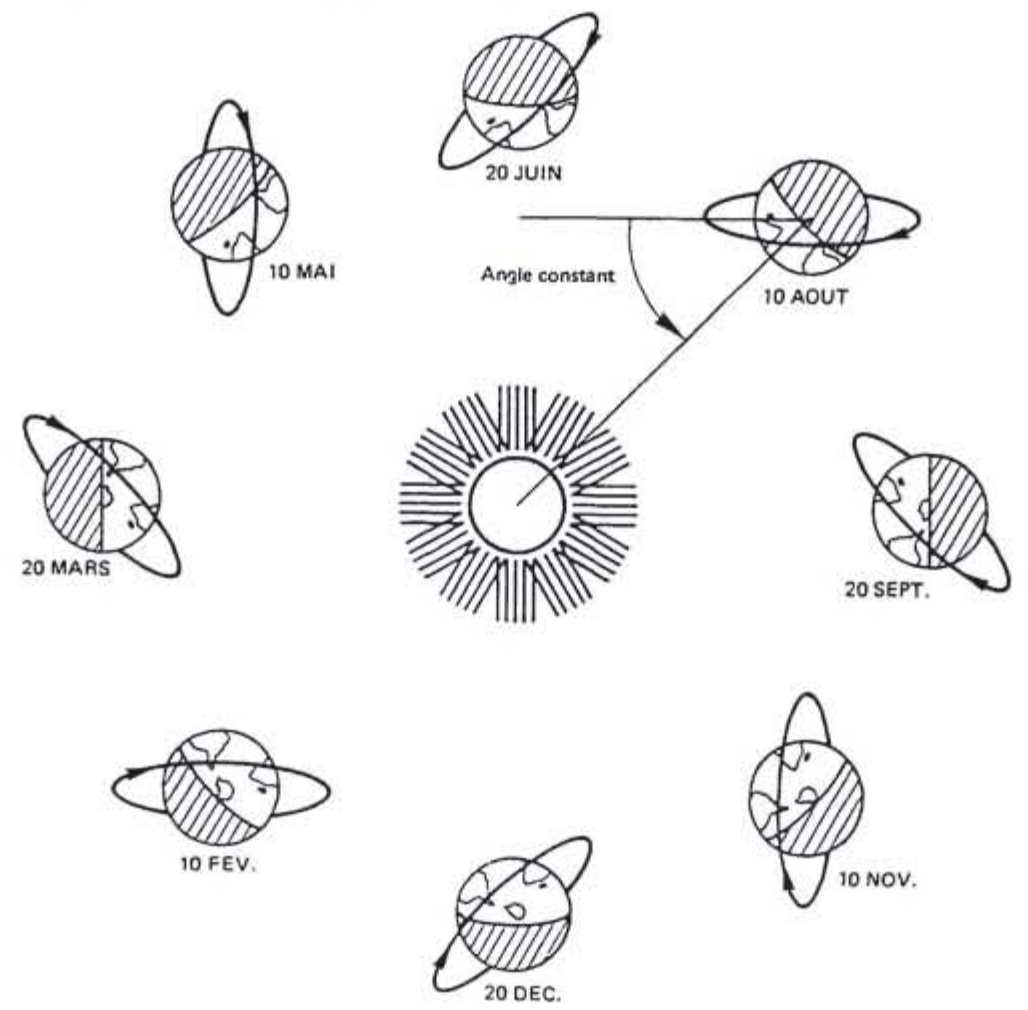

Figure 3 : Variation du plan d'orbite au cours de la rotation de la Terre autour du Soleil. 


\subsection{Le satellite}

Le satellite est composé d'une charge utile et d'une plate-forme multimissions. La charge utile est constituée des deux instruments HRV, d'un appareil d'enregistrement des données sur bande magnétique, et d'un ensemble de transmission vers le sol. La plate-forme assure les fonctions de servitude nécessaires à l'accomplissement de la mission : le maintien précis de l'orbite, la stabilisation 3 axes, l'alimentation électrique et le contrôle thermique, la télémesure de maintenance a $2 \mathrm{GHz}$, et la programmation de la charge utile par un calculateur de bord dont la mémoire est chargée par télécommande.

La stabilisation trois axes est assurée par des gyroscopes qui mesurent les dérives de vitesse du satellite dont les fréquences sont inférieures a $2 \mathrm{~Hz}$, par un senseur solaire et un senseur de visée du limbe terrestre. Ces systèmes de mesure permettent d'assurer un pointage de l'instrument à mieux que $0.5^{\circ}$ environ, sur chacun des axes de tangage, roulis et lacet.

\section{4.'instrument HRV}

Le principe de la prise de vue est un balayage par une ligne constituée par 3000 photodétecteurs (mode multibande) ou 6000 (mode panchromatique) placée perpendiculairement au vecteur vitesse instantané du satellite. Ce principe d'analyse d'image désigné par un terme anglais "Push - Broom", est intermédiaire entre une analyse globale d'image (ex. photographie) et l'analyse séquentielle à deux dimensions par un détecteur unique.

Le schéma optique de l'instrument [Refs. 4, 5] apparait en Figure 4. On décrit successivement les diverses composantes de la chaîne opto-électronique instrumentale.

Le télescope, de focale 1.08 mètre et de diamètre de pupille d'entrée $33 \mathrm{~cm}$, est une combinaison catadioptrique à miroir sphérique, dérivée du télescope de Schmidt à correction sphérique. Cette combinaison a été choisie pour ses performances en résolution, sa très grande tolérance

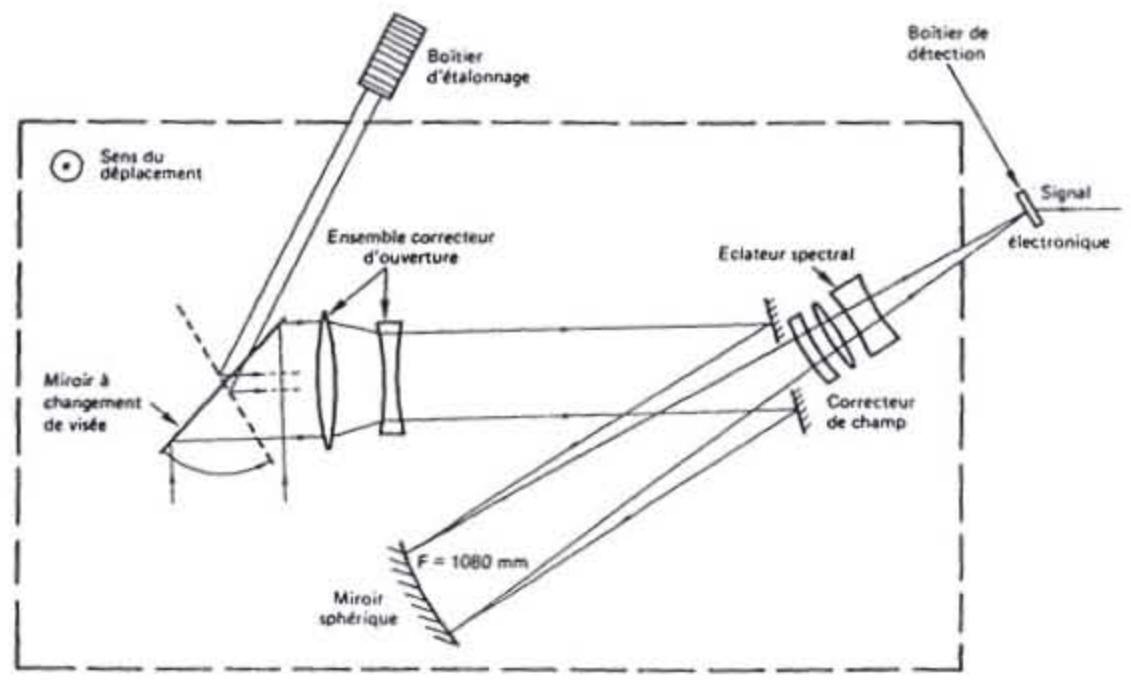

Figure 4 : Décomposition fonctionnelle du système optique. 
aux déformations mécaniques et thermiques, et pour son bon comportement chromatique. Le télescope possède un ensemble correcteur de champ dont la position longitudinale peut être ajustée. La mise au point la plus favorable, pour l'ensemble des quatre bandes utiles, est obtenue par déplacement du correcteur de champ. Le réglage effectué avant lancement peut être retouché lors des opérations en orbite (refocalisation en vol).

Au sein du boîtier de détection, le séparateur spectral (Figure 5) est constitué par un train de lames prismatiques sur lesquelles sont disposés des filtres dichroïques.

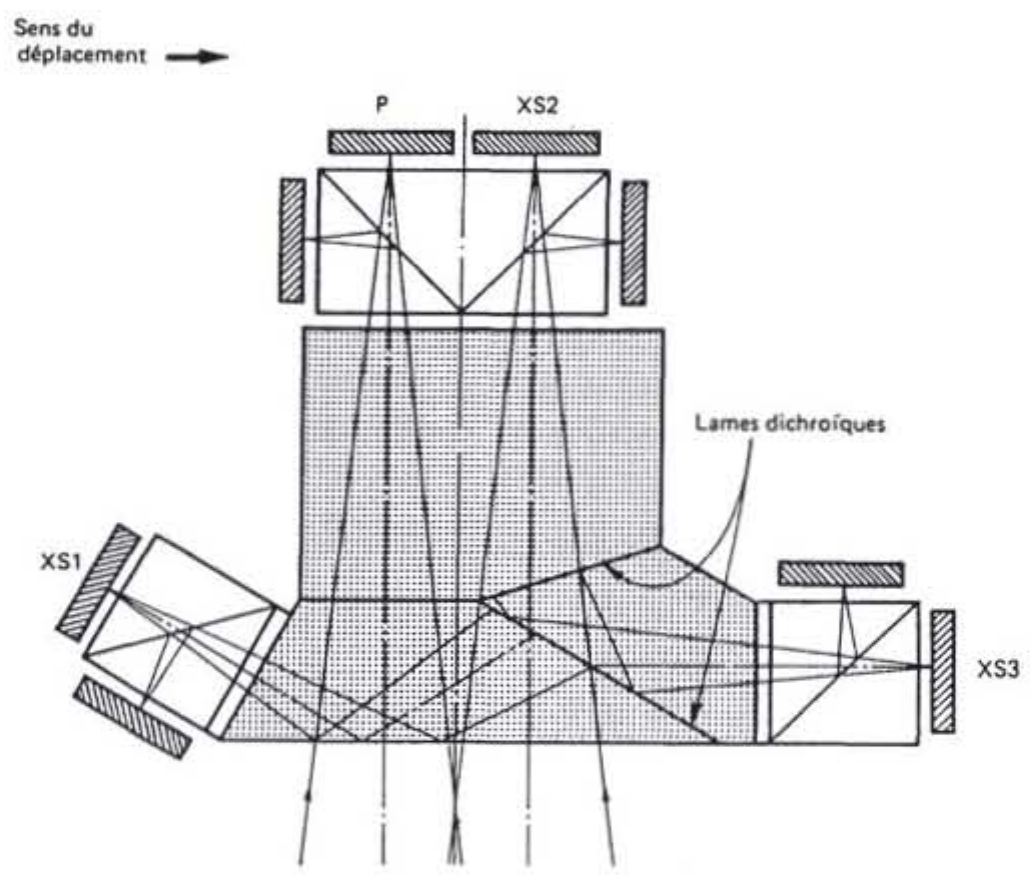

Figure 5 : Principe de la séparation spectrale.

Les quatre lignes de détecteurs constituées chacune par quatre barrettes CCD de 1728 pixels de 13 microns sont superposées optiquement avec une grande précision dans chaque plan focal. Les détecteurs CCD travaillent à la température de $20^{\circ} \mathrm{C}$.

L'assemblage des quatre barrettes d'une ligne de détecteurs est réalisé optiquement par un cube séparateur (lame semi) appelé "divoli" (diviseur optique de ligne) (Figure 6). Les "divolis" donnent des lignes pratiquement superposables. Dans cet assemblage, seuls 1500 détecteurs par barrette sont utilisés sur les 1728 disponibles. Pour les trois lignes de détecteurs obtenues en mode multibande, on additionne les signaux de deux détecteurs consécutifs, de façon à obtenir 3000 éléments par ligne, au lieu de 6000.

A chacune des 4 bandes spectrales est associée une voie d'amplification du signal électrique fourni par les détecteurs, qui comprend 8 valeurs discrètes de gain étagées selon une progression géométrique de raison 1,3. La numérisation est réalisée suivant un codage linéaire sur 8 bits en mode multibande, et suivant un codage de type DPCM 8-5-5-8 sur 6 bits en mode panchromatique. 


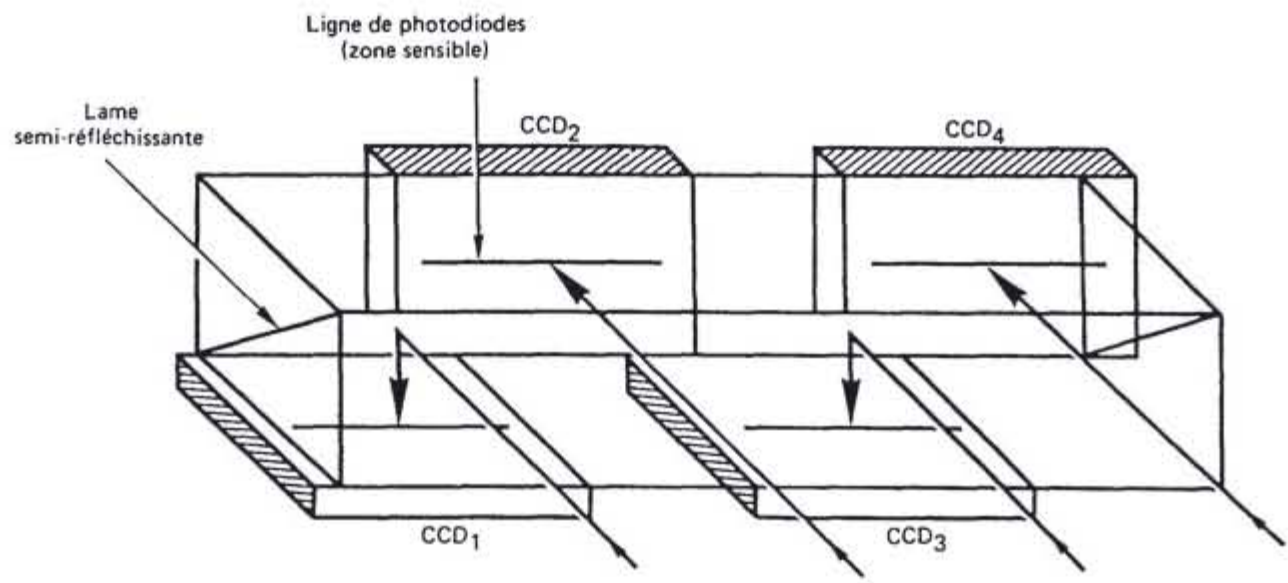

Figure 6: Montage des 4 barrettes de détecteurs sur un divoli.

Chaque instrument HRV est muni d'un boîtier d'étalonnage permettant d'éclairer le boîtier de détection à travers l'instrument HRV, grâce à un positionnement adéquat du miroir à changement de visée (Figure 4). Deux sources lumineuses peuvent être utilisées (Figure 7):

- Une lampe étalonnée contenue dans le boîtier d'étalonnage et susceptible d'éclairer l'ensemble des détecteurs. Le système comprend une lampe halogène de $20 \mathrm{~W}$ à filament de tungstène, placée à l'intérieur d'un montage de Koelher qui renvoie à l'infini l'image d'un plan uniformément éclairé. Ce système, placé à l'extérieur du télescope, envoie un faisceau lumineux à travers le télescope par l'intermédiaire du miroir à changement de visée de façon à éclairer uniformément (ou plutôt suivant un profil connu) toute la ligne de détecteurs.

- Un capteur solaire à fibres optiques permettant d'éclairer seulement quelques détecteurs dans chaque bande spectrale à l'aide du rayonnement solaire recueilli à l'extérieur. Du côté éclairé par le soleil, les fibres sont disposées en trois paquets distincts de façon à recevoir l'éclairement solaire dans les mêmes conditions, quelle que soit la période de l'année, le satellite étant en sortie d'éclipse.

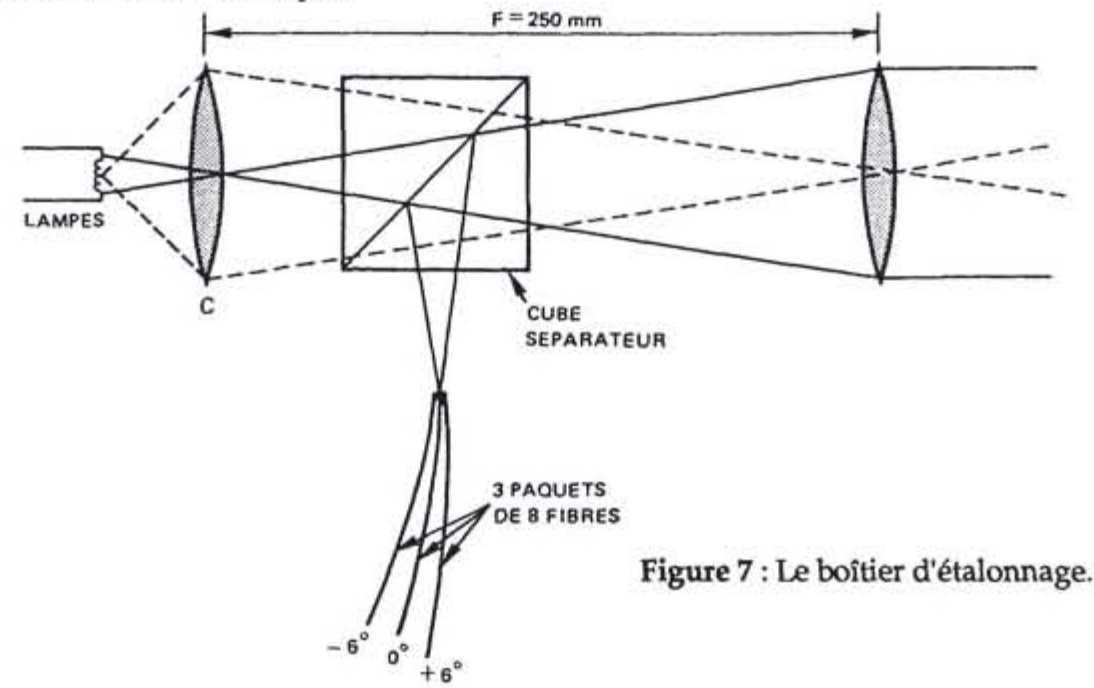




\subsection{Installations au sol et produits}

Deux stations au sol, Toulouse et Kiruna, sont utilisées pour recueillir la télémesure directe et enregistrée du satellite. Un grand nombre de stations de réception directe ont par ailleurs été construites de par le monde.

Les stations de Toulouse et Kiruna contiennent un centre d'archive et de prétraitement qui archive toutes les données brutes en y adjoignant une notation de couverture nuageuse, et qui fournit des produits (images utilisables) à la demande.

Le niveau de sophistication des prétraitements effectués pour réaliser ces produits est variable. Par exemple, le produit de niveau 0 est un produit d'échange, avec une radiométrie brute et des données auxiliaires permettant d'effectuer des traitements radiométriques ultérieurs. Le produit de niveau 1B contient une radiométrie corrigée des sensibilités des différents détecteurs et géométriquement superposable localement à une carte. D'autres produits de niveau plus élevé, comprenant notamment des corrections géométriques très précises utilisant des prises de points d'appui peuvent également être réalisés.

\section{LA QUALITE DES IMAGES}

On passe en revue dans ce paragraphe les principaux critères de qualité des images directement associées au concept instrumental de SPOT - dynamique des images, résolution radiométrique et spatiale, superposabilité multispectrale - puis, dans un second temps, les principales méthodes utilisées pour l'étalonnage radiométrique de l'instrument HRV [Réf. 6].

\subsection{Dynamique}

Le problème de l'adaptation des gains instrumentaux à la dynamique du signal incident est assez délicat. En effet, la luminance en entrée de l'instrument L, qui est la quantité physique mesurée par l'instrument, dans une bande spectrale donnée, est reliée à la réflectance $\rho$ des paysages observés (Terre + atmosphère), par une relation du type :

$$
\mathrm{L}=\frac{1}{\pi} \rho \mathrm{E}_{\mathrm{S}} \cos \theta_{\mathrm{S}},
$$

où $\mathrm{E}_{\mathrm{S}}$ est l'éclairement spectral solaire et $\boldsymbol{\theta}_{\mathrm{s}}$ l'angle zénithal solaire. La réflectance $\rho$, caractéristique des paysages observés, peut être fortement variable d'un paysage à l'autre. Par exemple, dans les bandes visibles, elle a pour valeur environ 0,05 pour la végétation active, de l'ordre de $0,2-0,3$ sur des sites urbains ou des sites désertiques, et pratiquement 1 sur de la neige fraîche.

Par ailleurs, la valeur de $\cos \theta_{\mathrm{s}}$ varie fortement, typiquement d'un facteur 4 , avec la latitude et avec la saison.

La luminance des paysages observables peut ainsi varier de façon très significative d'un paysage à l'autre. Sur SPOT 1, on a appliqué une logique d'utilisation à gain unique, dont la valeur a été conçue pour que le signal soit en limite de saturation ( 255 comptes numériques) sur des sites désertiques fortement réfléchissants $(\rho \sim 0,5)$ a l'équateur. Il en est résulté une dynamique de signal assez faible sur certaines zones peu réfléchissantes (par exemple, forêt dans le visible) et particulièrement en hiver.

La tendance développée actuellement, pour SPOT 2 et les satellites suivants, est de passer progressivement d'une utilisation monogain à une utilisation de gains multiples. 
Pour réaliser cette évolution, une base de données a été construite à partir de 1987, constituée de tous les histogrammes d'images SPOT acquises sans nuage dans les stations de Kiruna et Toulouse [Réf. 7]. Cette base de données permet de construire graduellement, en utilisant les coefficients d'étalonnage absolus de SPOT, une cartographie mondiale des réflectances des paysages tels qu'ils sont observés aux résolutions spatiale et spectrale des instruments HRV (Figure 8).

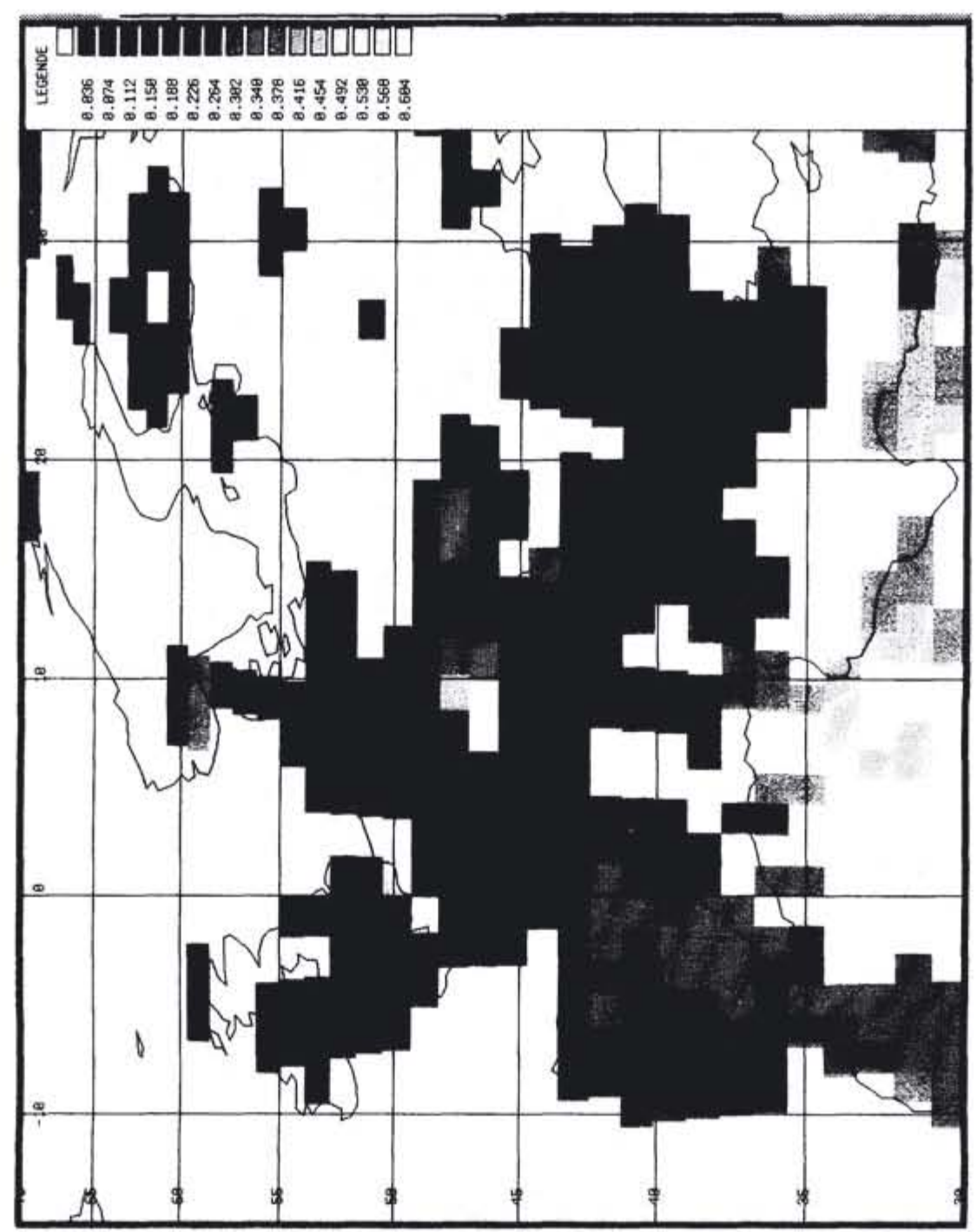

Figure 8: Cartographie des réflectances maximales observables dans la bande XS2 de SPOT 2. 
Les gains analogiques instrumentaux optimaux sur une région donnée, à une période donnée peuvent ainsi être conçus à l'avance en utilisant les résultats issus de la base de données d'histogrammes .

\subsection{Résolution radiométrique}

La performance de résolution radiométrique, c'est-à-dire la mesure du bruit sur une partie d'image (imagette) en visée d'un paysage uniforme, provient pour l'instrument à balayage Push-Broom qu'est HRV de deux sources de perturbations indépendantes : d'une part, le bruit provenant de chaque détecteur pris individuellement, qui génère des perturbations le long des colonnes de l'image, d'autre part les bruits de résidus d'égalisation interdétecteurs.

Le bruit lié au détecteur est mesuré en utilisant la bonne stabilité temporelle du signal émis par la lampe bord du boîtier d'étalonnage. Le rapport signal sur bruit obtenu, mesuré à une luminance environ moitié de la luminance de saturation du système, est de l'ordre de 250 à 400 suivant les bandes, comme l'indique le Tableau 2.

\begin{tabular}{|l|c|c|c|c|c|c|c|c|}
\hline & \multicolumn{4}{|c|}{ HRV1 } & \multicolumn{4}{c|}{ HRV2 } \\
\cline { 2 - 9 } & PA & XS1 & XS2 & XS3 & PA & XS1 & XS2 & XS3 \\
\hline 7 mars 1990 & 285 & 303 & 266 & 394 & 311 & 300 & 260 & 381 \\
\hline 8 novembre 1990 & 273 & 295 & 259 & 383 & 299 & 285 & 246 & 374 \\
\hline
\end{tabular}

Tableau 2 - Rapport signal/bruit le long des colonnes de l'image pour SPOT 2 à deux dates différentes

La variation du bruit en fonction de la luminance ne peut être mesurée directement en vol ; on en obtient cependant une estimation en recalant les mesures faites au sol, pour différentes luminances, avec la mesure faite en vol à une luminance unique [Réf. 8].

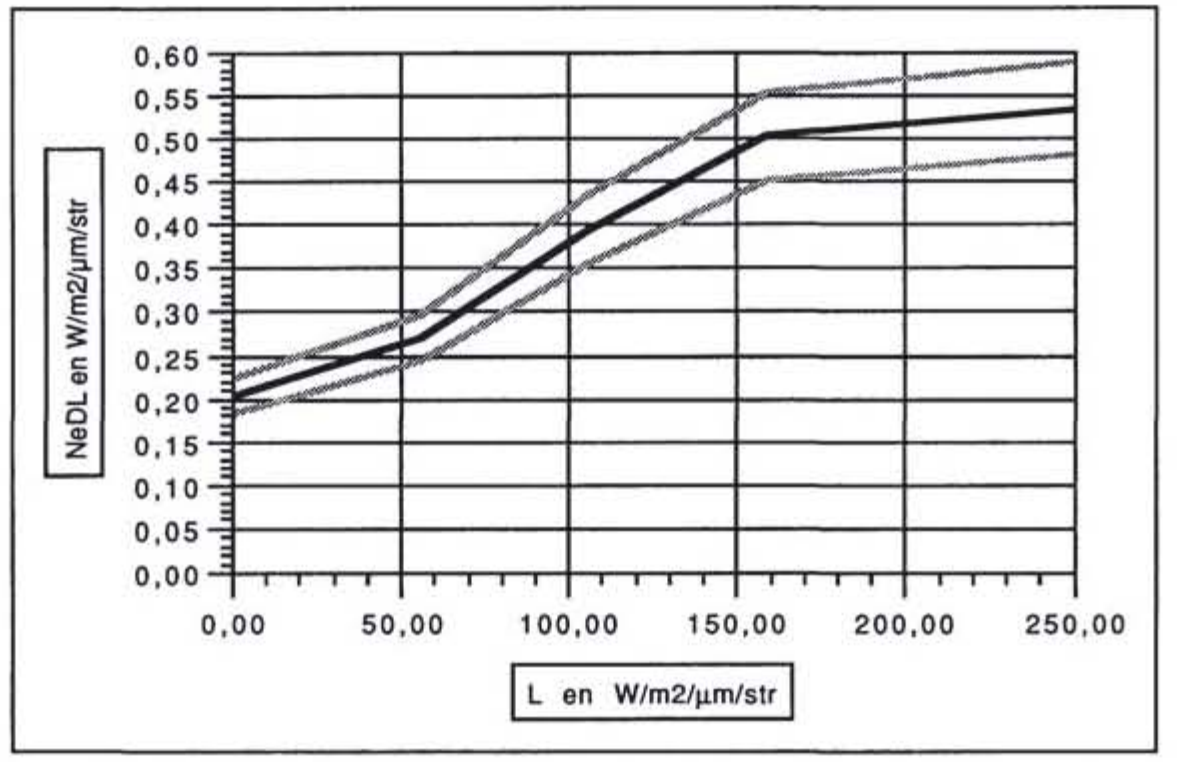

Figure 9: Le bruit détecteur SPOT $2 \mathrm{~Pa}$. Les traits grisés représentent des barres d'erreur. 
La Figure 9 représente ainsi un exemple de gabarit de bruit obtenu pour les bandes panchromatiques de SPOT 2. Notons par ailleurs qu'en première approximation le bruit détecteur peut être considéré comme blanc et gaussien.

Le bruit de résidus d'égalisation provient du fait que chacun des eléments d'une ligne de détecteurs CCD a une sensibilité au rayonnement et un signal d'obscurité qui lui sont propres, et que le processus de correction de ces effets peut ne pas être parfait.

Les notions de sensibilité au rayonnement et de signal d'obscurité sont définies précisément au moyen du modele radiométrique de prise de vue instrumentale, qui relie dans une bande spectrale donnée, le compte numérique x en sortie de l'instrument à la luminance L observée en entrée de l'instrument. Ce modèle s'écrit :

$$
x_{l p}=A_{p} L+C_{p}
$$

où 1 et p désignent la $l^{\text {ième }}$ acquisition du détecteur $\mathrm{p}, \mathrm{A}$ est le coefficient d'étalonnage absolu, $\mathrm{g}_{\mathrm{p}}$ s'appelle le coefficient d'égalisation interdétecteur du détecteur $\mathrm{p}$, et $\mathrm{C}_{\mathrm{p}}$ est le signal d'obscurité du détecteur p. Dans l'équation (2), L représente la luminance équivalente à la luminance spectrale $L(\lambda)$ dans la bande spectrale considérée, de profil de sensibilité spectrale $S(\lambda)$,

$$
L=\frac{\int L(\lambda) S(\lambda) d \lambda}{\int S(\lambda) d \lambda}
$$

L'instrument HRV est tel que $C_{p}$ peut atteindre quelques comptes numériques, et que $g_{p^{\prime}}$ défini de façon à ce que la moyenne de $\mathrm{g}_{\mathrm{p}}$ sur l'ensemble des détecteurs $\mathrm{p}$ soit égale à 1, peut atteindre des valeurs de 0,9 a 1,1 environ. Ces différents coefficients sont mesurés (voir § 3.5.2) et on tient compte de ces mesures pour corriger radiométriquement l'image en suivant le modèle (2).

Néanmoins, après correction, des résidus d'égalisation peuvent apparaître, dus à des imprécisions de mesure des coefficients, et également au fait que la réalité instrumentale peut s'écarter légèrement du modèle radiométrique associé (2). La signature des résidus d'égalisation est aisément identifiable sur les images et se caractérise par la présence de structures ou de rayures verticales.

La mesure des bruits de résidus d'égalisation peut être effectuée sur les imagettes de paysages neigeux de très bonne uniformité observables dans les régions polaires. On peut alors réaliser une courbe type de bruits d'égalisation en fonction de la luminance à partir d'une extrapolation de ces mesures [Réf. 8], comme le montre par exemple la Figure 10 pour la bande panchromatique de SPOT 2.

En sommant (quadratiquement) ces derniers résultats avec ceux obtenus pour le bruit détecteur, on obtient la performance globale de résolution radiométrique NeDL sur des imagettes SPOT en fonction de la luminance $L$ d'observation. On peut alors remonter a la notion de variation minimum de réflectance au sol détectable NeD $\rho$ par l'instrument HRV, pour un angle zénithal solaire $\theta_{\mathrm{S}}$ donné. Ce calcul ne peut être fait qu'en utilisant une modélisation des effets atmosphériques sur le signal satellitaire. A titre d'exemple, les valeurs de NeD $\rho$ obtenues sur SPOT 1 pour $\theta_{\mathrm{S}}=15^{\circ}$ et $\theta_{\mathrm{S}}=60^{\circ}$ apparaissent dans le Tableau 3. 


\begin{tabular}{|c|c|c|c|c|}
\hline & \multicolumn{2}{|c|}{ HRV1 } & \multicolumn{2}{c|}{ HRV2 } \\
\hline & $\theta_{\mathrm{s}}=15^{\circ}$ & $\theta_{\mathrm{s}}=60^{\circ}$ & $\theta_{\mathrm{s}}=15^{\circ}$ & $\theta_{\mathrm{s}}=60^{\circ}$ \\
\hline $\mathrm{Pa}$ & 0.0019 & 0.0038 & 0.0019 & 0.0038 \\
\hline $\mathrm{Xs}_{\mathrm{N} 1}$ & 0.0014 & 0.0029 & 0.0014 & 0.0029 \\
\hline $\mathrm{Xs}_{\mathrm{N} 2}$ & 0.0020 & 0.0042 & 0.0018 & 0.0036 \\
\hline $\mathrm{Xs}_{\mathrm{N} 3}$ & 0.0015 & 0.0031 & 0.0015 & 0.0031 \\
\hline
\end{tabular}

Tableau 3 - Valeurs de NeD $\rho$ sur une imagette obtenue pour SPOT 1 à deux angles $\theta_{\mathrm{s}}$ diférents

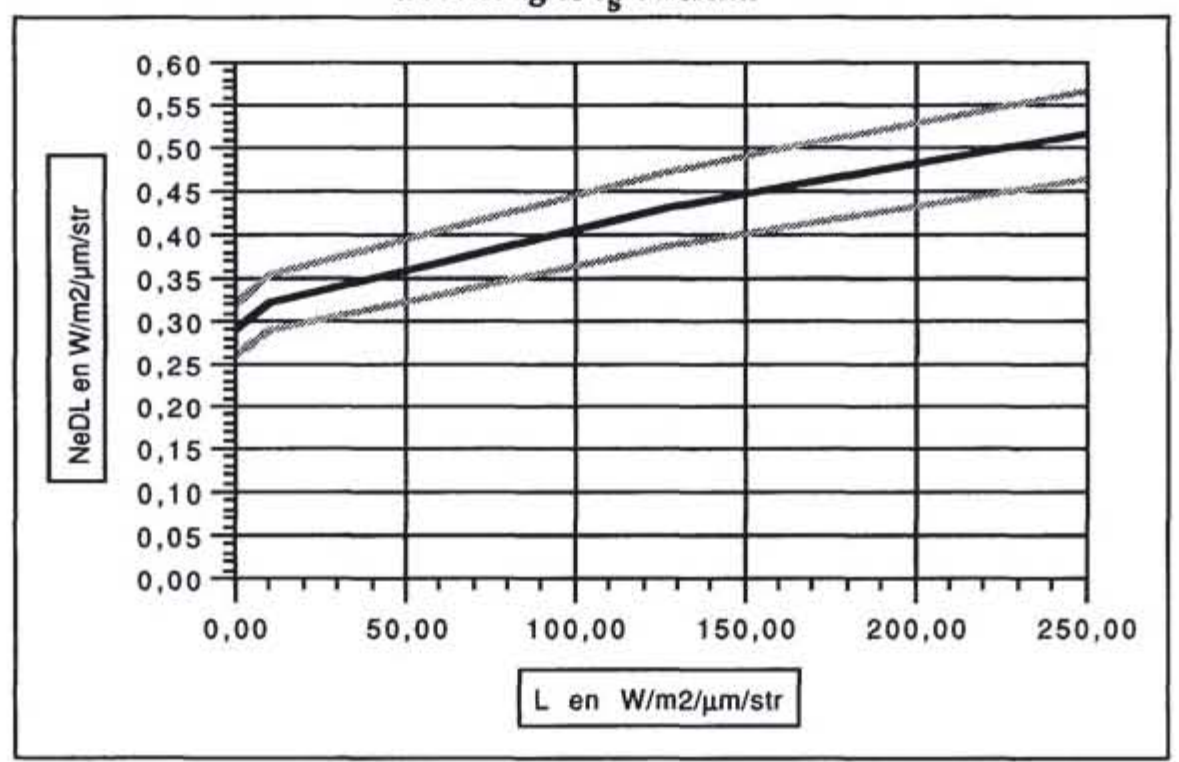

Figure 10 : Le bruit de résidu d'égalisation SPOT 2 Pa.

Les traits grisés représentent des barres d'erreur.

Les résultats qui précèdent sont ce qu'on pourrait appeler des résultats nominaux. Toutefois, un certain nombre d'images présentent des défauts particuliers, qui viennent dégrader parfois sensiblement la résolution radiométrique, et qui nécessitent des traitements supplémentaires pour être corrigés.

Le premier de ces défauts apparaît sur certains sites désertiques de haute réflectance, sous la forme, dans la bande verte XS1, de 4 bandes verticales correspondant aux quatre barrettes de la ligne de détecteurs. Les écarts radiométriques entre bandes peuvent atteindre trois comptes numériques. Ce défaut provient du fait que le "divoli" introduit, dans la bande XS1, des différences significatives de sensibilité spectrale $S(\lambda)$ entre barrettes vues en transmission et barrettes vues en réflexion. Ces différences prennent de l'importance quand la répartition 
spectrale de réflectance $\rho(\lambda)$ des sites observés diffère sensiblement de celle des paysages utilisés pour la mesure des $g_{\mathrm{p}}$ (voir § 3.5.2), ce qui est le cas des sols désertiques.

D'autres défauts sont visibles sur certaines images de faible réflectance, comme la forêt dans le visible et la mer.

Ces défauts, dont l'amplitude peut atteindre deux comptes numériques, proviennent d'instabilités temporelles à court terme ( $\sim 10$ minutes) des courants d'obscurité, et peuvent provenir également, pour une faible part, de défauts de linéarité des détecteurs à faible luminance.

D'une manière générale, la structure spatiale de ces défauts est bien marquée (bandes ou rayures verticales), ce qui permet d'élaborer des algorithmes relativement simples de traitement et de correction au sol des défauts.

\subsection{Résolution spatiale}

La performance de résolution spatiale est liée d'une part au pas d'échantillonnage ( $10 \mathrm{~m}$ ou 20 $\mathrm{m}$ au nadir suivant les modes spectraux) et d'autre part à la Fonction de Transfert de Modula ion (FTM), transformée de Fourier de la réponse impulsionnelle du système de prise de vue.

Les principaux effets intervenant dans la performance de FTM sont les suivants :

- la diffraction résultant de l'ouverture finie du télescope et de l'occultation d'une partie du rayonnement incident par le trou central du premier miroir plan du télescope,

- l'optique du séparateur spectral dégrade également un peu les contrastes,

- la surface finie des détecteurs élémentaires,

- la diaphotie, c'est-à-dire le phénomène physique de diffusion d'une partie des charges contenues dans un détecteur donné vers les détecteurs voisins; cet effet ne joue que suivant les lignes de l'image,

- l'effet de filé dâ au temps d'exposition non nul et au déplacement du point visé pendant ce temps d'exposition; cet effet n'intervient que le long des colonnes de l'image.

On peut, en analysant chacun de ces effets et en les paramétrant, réaliser une modélisation de la FTM dont les coefficients sont ajustés pour recouper au mieux les résultats de mesure obtenus au sol sur un banc d'essai avant tir. A titre d'exemple, la FTM de la bande panchromatique de SPOT 2 peut être modélisée de la façon suivante [Réf. 9] :

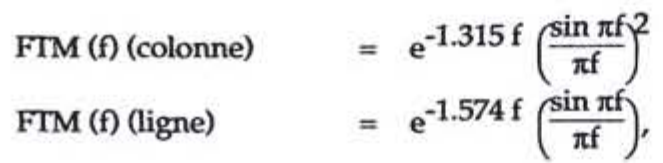

où $\mathrm{f}$ désigne le rapport entre la fréquence spatiale et la fréquence spatiale d'échantillonnage $\mathrm{f}_{\mathrm{e}}$. Les courbes correspondantes de FTM sont tracées en Figure 11.

Des modélisations analogues peuvent également être faites pour les autres bandes spectrales de SPOT ; on donne ici simplement, dans le Tableau 4, les valeurs de FTM de SPOT 2 mesurées avant tir à la fréquence de Nyquist $f_{e} / 2$. 


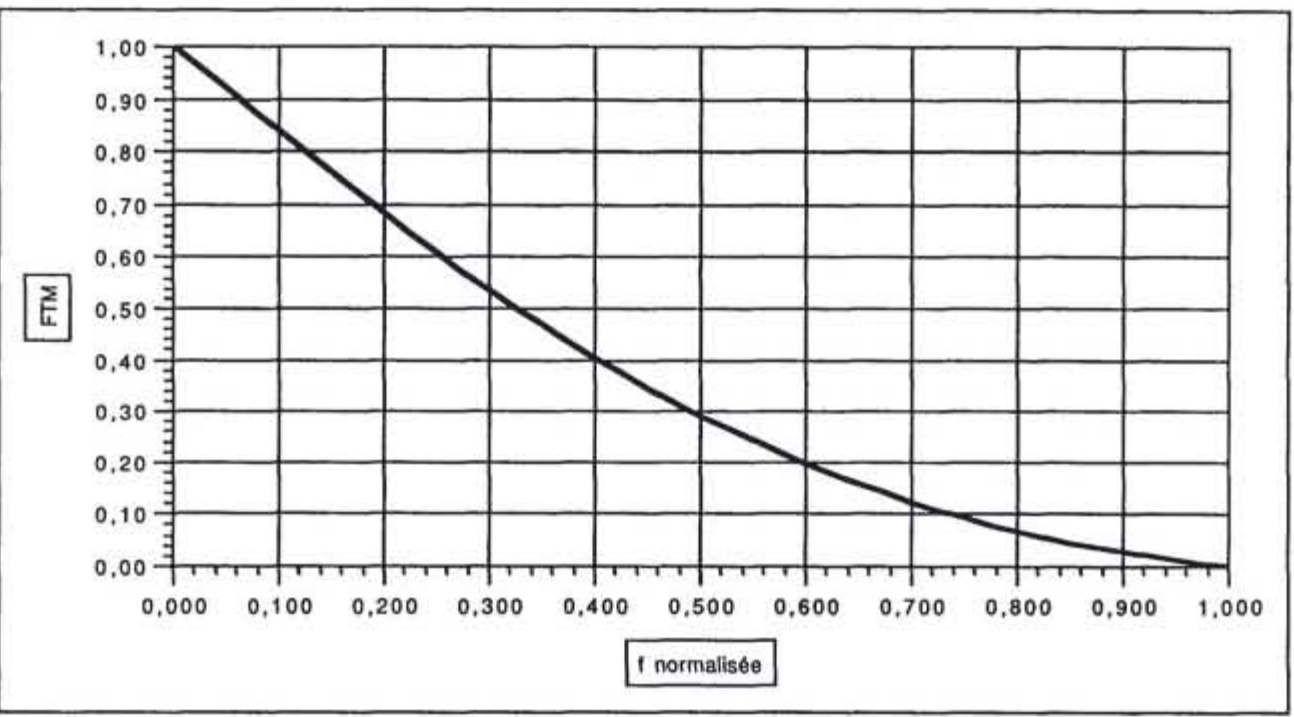

Figure 11 a : Gabarit de FTM ligne SPOT $2 \mathrm{~Pa}$

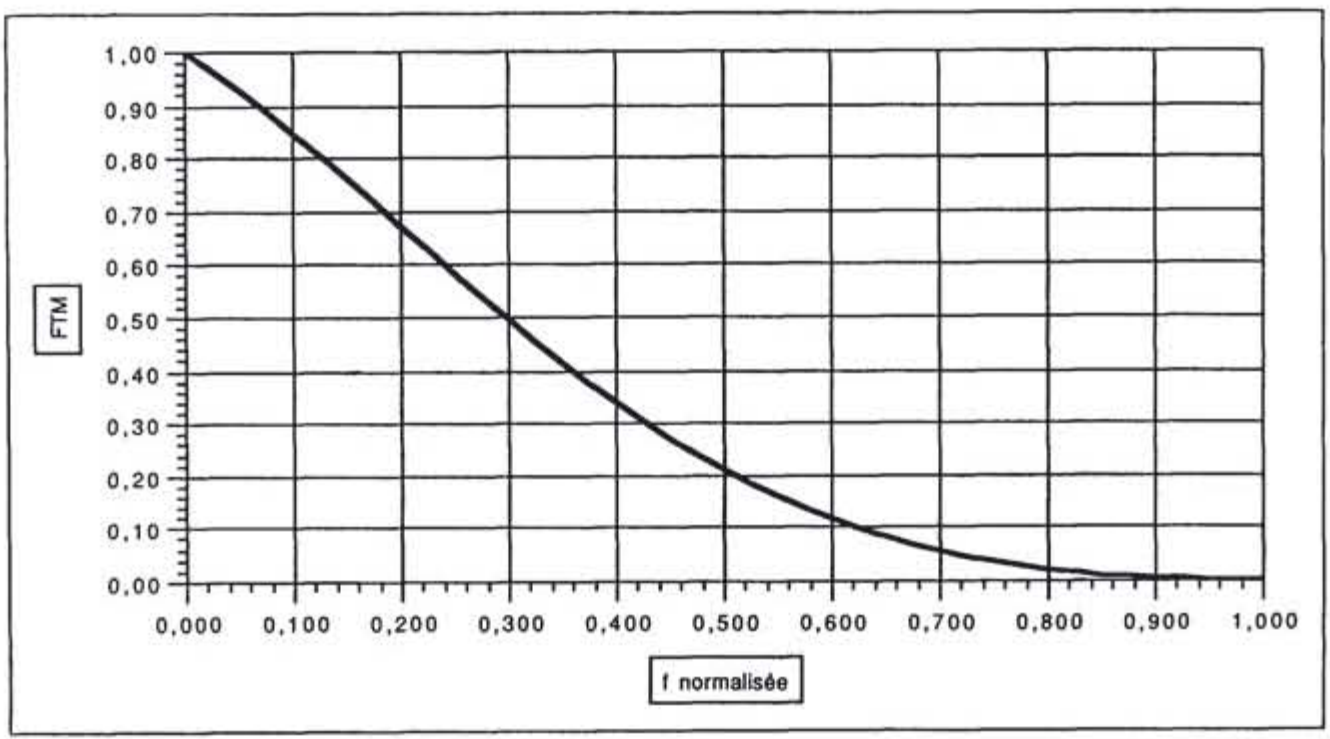

Figure 11 b : Gabarit de FTM colonne SPOT $2 \mathrm{~Pa}$ 


\begin{tabular}{|c|l|l|l|}
\hline BANDE & FTM & HRV1 & HRV2 \\
\hline \multirow{2}{*}{$\mathrm{Pa}$} & Colonne & 0,21 & 0,22 \\
& Ligne & 0,27 & 0,30 \\
\hline \multirow{2}{*}{ XS1 } & Colonne & 0,42 & 0,42 \\
& Ligne & 0,48 & 0,50 \\
\hline \multirow{2}{*}{ XS2 } & Colonne & 0,42 & 0,42 \\
& Ligne & 0,44 & 0,47 \\
\hline \multirow{2}{*}{ XS3 } & Colonne & 0,40 & 0,41 \\
& Ligne & 0,37 & 0,39 \\
\hline
\end{tabular}

Tableau 4 - Valeurs de FTM à fe/2 mesurées avant tir sur SPOT 2

\subsection{Superposabilité multispectrale}

Les trois bandes spectrales du mode multibandes doivent avoir une très bonne qualité de superposition. Rappelons que les 3000 photoéléments d'une bande spectrale XS sont constitués de quatre barrettes $C C D$ de 1728 détecteurs, dont on sélectionne seulement 1500 utiles et où l'on couple deux détecteurs adjacents pour réaliser un photoélément. Les défauts de positionnement des quatre barrettes, la superposition optique imparfaite des trois bandes spectrales due aux défauts du télescope et aux problèmes de métrologie et d'assemblage des divolis sur le séparateur spectral, font que les centres géométriques des trois photoéléments homologues dans les trois bandes spectrales ne sont pas exactement superposables. Les résultats obtenus montrent des défauts de type "basse fréquence" d'amplitude 0,1 pixel environ et des cassures aux interbarrettes également de l'ordre de 0,1 pixel.

Globalement, le rayon du cercle au sol incluant les détections de visée de trois photoéléments homologues dans les trois bandes spectrales reste inférieur à 0,15 pixel.

\subsection{Etalonnage radiométrique}

\subsubsection{Etalonnage absolu}

L'étalonnage absolu consiste à mesurer le coefficient $\mathrm{A}$ du modèle radiométrique de prise de vue (Eq. (2)) avec la précision la meilleure possible, et ce, tout au long de la durée de vie du satellite. Pour atteindre cet objectif, un certain nombre de mesures indépendantes ont été mises en place [Réf. 10] :

(i) Les coefficients A sont mesurés avant tir par utilisation d'une sphère intégrante étalonnée de diamètre extérieur $1,60 \mathrm{~m}$, dont la pupille de sortie couvre la totalité de la pupille d'entrée du HRV. Les valeurs trouvées sont ensuite corrigées des pertes de transmission lors du passage au vide, par des mesures de sensibilité spectrale faites à l'air ambiant et ensuite sous vide. En effet, les filtres dichroïques utilisés dans HRV ont un profil plus étroit sous vide qu'à l'ambiante, le passage sous vide provoquant la résorption de l'eau contenue dans les filtres et modifiant ainsi leurs caractéristiques spectrales. Les pertes de transmission air-vide peuvent atteindre 10 à $20 \%$. 
(ii) Des mesures sont effectuées en vol sur le site d'étalonnage de White Sands (NouveauMexique). Celles-ci consistent à mesurer, à l'aide de radiomètres de terrain, la réflectance d'une portion de ce site ainsi que l'épaisseur optique et la nature des aérosols de l'atmosphère. L'application d'un code de transfert radiatif atmosphérique permet alors de déduire la luminance observable du site au-dessus de l'atmosphère. La comparaison avec l'image de SPOT prise au même moment que les vérités-terrain fournit les coefficients d'étalonnage recherchés.

Des campagnes de ce type sont organisées environ une fois tous les six mois, en collaboration avec l'équipe du Pr. Philip Slater de l'Université de l'Arizona.

(iii) Le suivi temporel du signal moyen délivré par la lampe d'étalonnage bord est une bonne mesure de l'évolution temporelle de l'étalonnage du système, à condition que la lampe soit stable dans le temps. Le recoupement des différentes méthodes d'étalonnage entre elles permet d'affirmer que la stabilité sur quatre ans du signal lampe de SPOT 1 a été meilleure que $2 \%$. La Figure 12, qui représente les pertes de transmission instrumentale mesurées par la lampe de SPOT 1 sur une durée de quatre ans, montre que ces pertes peuvent atteindre de $13 \%$ \ $25 \%$ suivant les bandes spectrales.

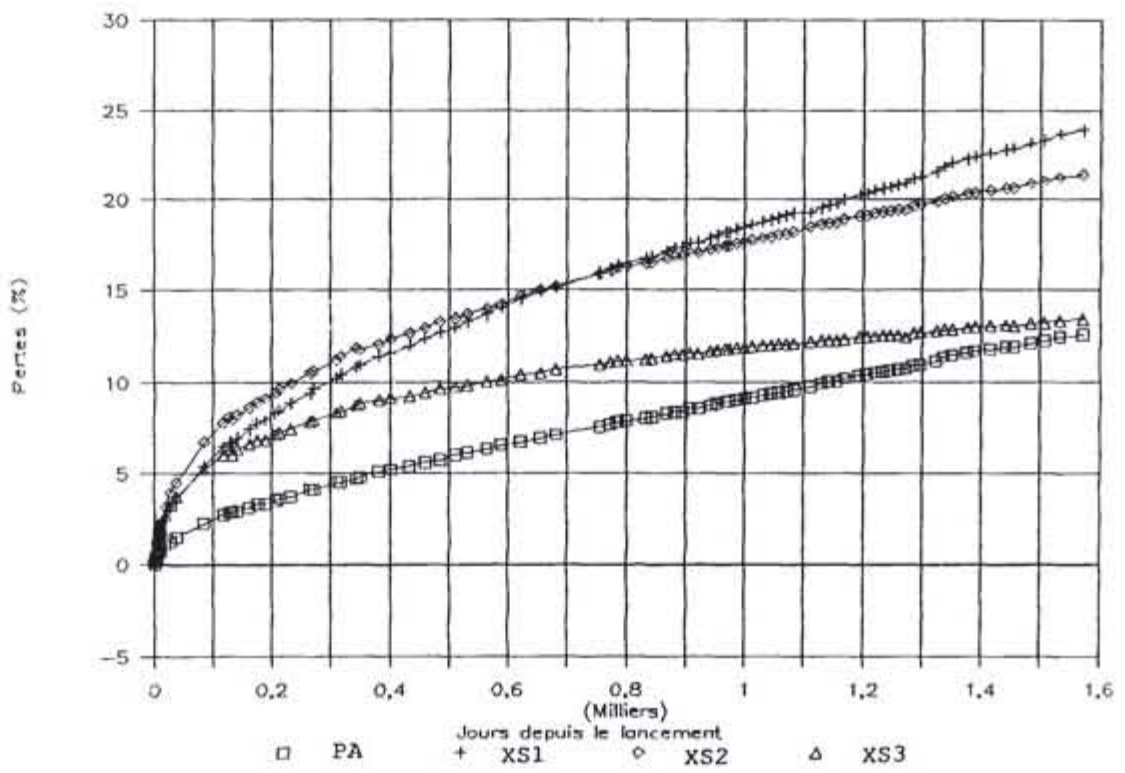

Figure 12 : Pertes de transmission mesurées par la lampe en 4 ans de durée de vie pour l'instrument HRV2 de SPOT 1

(iv) Le suivi temporel du signal du capteur solaire fournit une indication supplémentaire précieuse. La fonction du capteur solaire est, outre le suivi temporel, de faire une mesure absolue de l'étalonnage du système. Néanmoins, par suite de difficultés de caractérisation avant tir des transmissions du capteur solaire, et de difficultés de mise au point de logiciels opérationnels de traitement des informations fournies par ce capteur, ce système d'étalonnage n'a pas encore atteint son point de fonctionnement optimal.

(v) L'interétalonnage des deux instruments HRV est obtenu en comparant les histogrammes dans les bandes homologues de zones visées simultanément par les deux instruments. 


\begin{tabular}{|l|l|}
\hline Etalornage absolu & $6 \%$ \\
Etalonnage multidate & $2 \%$ \\
Etalonnage interbande & $3 \%$ \\
Etalonnage inter HRV & $2 \%$ \\
\hline
\end{tabular}

\section{Tableau 5 - Précision d'étalonnage obtenue}

L'ensemble de ces méthodes, et leur analyse comparée, permet d'estimer avec une précision satisfaisante l'étalonnage de système, et de communiquer aux utilisateurs les résultats correspondants [Réfs. 11,12]. Les précisions estimées des coefficients fournis, suivant différents critères, sont expicitées dans le Tableau 5.

3.5.2 Egalisation des détecteurs

Dans son principe, le montage Koehler du boítier d'étalonnage bord permet au signal de la lampe d'avoir une bonne uniformité spatiale en entrée du HRV. Ce système est conçu de façon à suivre les variations temporelles des coefficients $g_{p}$ et à estimer leurs valeurs à un instant dofiné, à partir d'un jeu de coefficients $g_{p}$ connus à un instant initial.

Ce système n'a cependant pas pu être utilisé sur SPOT 1 parce que l'on s'est aperçu que le profil spatial du signal lampe n'est pas stable d'une expérience à l'autre. La différence de deux images lampe consécutives contient des pics d'une amplitude de plusieurs pour cent. Ces pics proviennent du fait qu'un quelconque petit défaut (micro-poussière, rayure) apparaissant sur la première lentille du montage de Koehler (Figure 7) est naturellement projeté sur les détecteurs en passant par le miroir à changement de visée. La reproductibilité de positionnement du miroir n'étant pas meilleure que $10^{-4}$ radian, un déplacement aléatoire du petit défaut considéré a lieu sur les détecteurs, de l'ordre de plusieurs pixels, ce qui rend impossible la mesure des coefficients d'égalisation.

Le boítier d'étalonnage a donc été légèrement modifié sur SPOT 2 pour éviter ce type de problème, d'une part en défocalisant légèrement la première lentille du montage de Koehler par rapport à la seconde, et d'autre part en rajoutant un verre dépoli entre la lampe et la première lentille de façon à couvrir l'ensemble de la pupille de sortie du boítier d'étalonnage et minimiser ainsi l'influence des micro-défauts présents sur la première lentille. Les résultats de mesure du boîtier d'étalonnage SPOT 2 sont actuellement en cours d'analyse.

La méthode opérationnelle d'égalisation des détecteurs a été sur SPOT 1, et continue d'être sur SPOT 2, l'utilisation de grandes étendues neigeuses de très bonne uniformité que l'on peut trouver dans les régions polaires du Groënland et de l'Antarctique. Le principe de la méthode est de considérer que sur ces étendues, chacun des détecteurs voit en moyenne sur une image la même luminance. L'analyse de la ligne moyenne de l'image fournit alors directement les coefficients d'égalisation $\mathrm{g}_{\mathrm{p}}$.

Des campagnes de mesure des coefficients $g_{p}$ sur les régions polaires ont lieu environ tous les six mois. C'est également à la même fréquence approximative que l'on réactualise les courants d'obscurité $\mathrm{C}_{\mathrm{p}}$, dont la mesure est comparativement bien plus facile, puisqu'il suffit pour cela de générer des images de nuit avec le miroir à changement de visée placé de façon à obturer le champ de vue. 
3.5.3 Mesure de la FTM en vol La méthode d'évaluation en vol de la FTM utilisée actuellement est essentiellement photointerprétative. Un catalogue d'images de référence simulant des images panchromatiques de SPOT est préparé avant tir. Ce catalogue est réalisé en utilisant des photographies aériennes, numérisées à la résolution de $3,33 \mathrm{~m}$, sur quelques villes du Sud de la France, dans une bande spectrale proche de la bande panchromatique. Une convolution paramétrée appliquée à l'image origine permet de simuler une FTM Gaussienne, $\mathrm{FTM}(\mathrm{f})=\exp \left(-\mathrm{kf}^{2}\right)$, où $\mathrm{k}$ est un paramètre ajustable. A partir d'une photographie d'origine, un jeu de neuf images rééchantillonnées à 10 mètres est réalisé, ayant neuf FTM différentes et décroissantes.

L'image de SPOT acquise en vol est alors comparée visuellement au catalogue d'images de référence, ce qui permet d'avoir une idée de la qualité de FTM obtenue en vol.

Des méthodes indirectes sont par ailleurs appliquées pour comparer les FTMs des deux instruments dans les bandes spectrales homologues, par une analyse du spectre fréquentiel des deux images d'une même zone visée simultanément par les deux instruments.

Notons que ces méthodes permettent de vérifier qu'il n'y a pas eu de dégradation importante entre les mesures avant tir et la réalité orbitale, mais sont insuffisantes à elles seules pour faire une évaluation complète en vol de l'ensemble de la courbe FTM (f). D'autres méthodes utilisant des mires au sol ou le vol simultané d'un capteur aéroporté, doivent être mises en place pour parvenir à cet objectif [Réfs 13,14]. Notons que l'étalonnage en vol de la fonction de transfert de modulation est important pour mettre au point des algorithmes performants de déconvolution et de restitution au sol des contrastes de l'image.

\section{CONCLUSIONS ET PERSPECTIVES}

On a, dans cet article, passé en revue les principales caractéristiques du système SPOT, en mettant plus particulièrement l'accent sur la description de l'optique instrumentale. On a également examiné les différents critères de qualité des images et les méthodes d'étalonnage correspondantes, en ne retenant dans cet examen que les critères de qualité image relevant directement de la conception de l'instrument HRV.

On peut évoquer ici très brièvement les critères de qualité géométrique des images, qui dépendent non seulement de l'instrument HRV, mais aussi de la précision avec laquelle on peut restituer l'orbitographie et l'attitude du satellite. Les performances géométriques de SPOT sont tout à fait remarquables. On est ainsi capable de localiser, sans prise de point d'appui, un pixel d'une image quelconque de SPOT 2 avec une précision en moyenne meilleure que 300 mètres, et au plus égale à 800 mètres. De même, l'erreur relative que l'on commet quand on mesure des distances sur une image corrigée géométriquement sans prise de point d'appui est inférieure à $10^{-3}$ sur les images de SPOT 1 et 2 . Enfin, la précision de restitution altimétrique et planimétrique lorsqu'on utilise des couples d'images stéréoscopiques est meilleure que 10 mètres.

En conclusion, on peut dire que la qualité des images SPOT est satisfaisante, et bien adaptée à ses différentes missions d'observation de la Terre. Le principe de balayage électronique "PushBroom" de l'image, qui a été une des originalités importantes du système SPOT, est dorénavant le principe de fonctionnement retenu par tous les systèmes optiques d'observation de la Terre à haute résolution géométrique qui verront le jour dans les dix ans qui viennent, en Europe et ailleurs. Le satellite SPOT 3, identique à SPOT 1 et 2, devrait être lancé en 1993 et le satellite SPOT 4, version améliorée du satellite SPOT comprenant une bande spectrale dans le moyen infrarouge, devrait prendre le relais de SPOT 3 vers 1997. Le radiomètre japonais AVNIR de la plate-forme ADEOS volera dès 1995 avec une résolution de 8 mètres. 
Une réflexion a d'ores et déjà été entreprise au CNES [Réf. 15] pour imaginer l'avenir de la filière SPOT, qui passe sans doute par une amélioration significative de la résolution spatiale. La conception optique de HRV a très vraisemblablement un potentiel d'évolution dans ce domaine et parait capable de s'adapter aux technologies de détecteurs CCD plus performants et plus petits ( 10 ou 7 microns) qui deviennent actuellement disponibles.

Remerciements: Je remercie G. OTRIO (CNES) pour d'utiles discussions sur la conception optique de l'instrument et $\mathrm{P}$. HENRY (CNES) pour une relecture critique du manuscrit.

\section{REFERENCES BIBLIOGRAPHIQUES}

[1] M. Courtois \& G. Weill , 1985, "The SPOT satellite system", Progress in Astronautics and Aeronautics series, vol. 97, published by the American Institute of Aeronautics and Astronautics, Abraham Schnapf Editor.

[2] Guide des Utilisateurs de Données SPOT - Document CNES et SPOT Image, 1986 - Disponible à SPOT Image.

[3] G. Bégni , Oct 1982, "Sélection of the optimum spectral bands for the SPOT satellite", Photogrammetric Engineering and Remote Sensing, vol 48., 1613-1620.

[4] JP. Deshayes et G. Otrio, Optical design of a high resolution multispectral scanner for Earth Observation, comptes rendus du 11è congrès CIO, Madrid, Sept. 1978.

[5] G. Otrio, SPOT, un observatoire spatial au service de la planète Terre, à paraître dans Opto, journal des anciens élèves de l'ESO.

[6] G. Bégni, B. Boissin et M. Leroy, SPOT Image Qualilty, Progress in Imaging Sensors, Proceedings ISPRS Symposium, Stuttgart, Sept. 1986, ESA SP-252.

[7] M. Dinguirard et P. Henry, Base de Données des Histogrammes SPOT, Proceedings ISPRS - 5 e colloque International de Mesures Physiques et Signatures en Télédétection, Courchevel, 1991.

[8] P. Henry, Modélisation radiométrique de la prise de vue SPOT-2 Pa à partir des résultats de recette en vol, Rapport interne CNES S2-NT-0B1-1404-CN, avril 1991.

[9] JM. Martinuzzi, Approximation analytique de la courbe de FTM Pa SPOT 2 en vue de la création d'un filtre de déconvolution 7 × 7, Rapport interne CNES S2-NT-0B1-1398-CN, mars 1991.

[10] M. Dinguirard, G. Begni et M. Leroy, SPOT 1 calibration results after 2 years of flight, SPIE-924, Recent Advances in Sensors, radiometry and data processing for Remote Sensing, Orlando, 1988.

[11] G. Bégni, SPOT Newsletters, Edition SPOT Image, 1987.

[12] P. Henry et M. Dinguirard, Document de synthèse sur l'étalonnage absolu des données SPOT, Rapport interne CNES S-NT-0B1-580-CN, février 1991, à paraître dans SPOT Newsletters.

[13] R. A. Schowengerdt, C. Archamety, R. C. Wrigley, Landsat Thematic Mapper Imaged-derived MTF, Photogrammetric Engineering and Remote Sensing, vol. 51, n 9, 1985.

[14] F. Robinet, D. Léger, H. Cerbelaud, S. Lafont, Obtaining the MTF of a CCD imaging system using an array of point sources : evaluation of performances, Proc. IGARSS'91, Helsinki, juin 1991.

[15] C. Fratter and JF. Reulet, SPOT 4 HRVIR instrument and future high resolution stereo instruments, SPIE Proceedings Vol. 1490, Orlando, 1991. 